\title{
A New Compression Intermediate Strain Rate Testing Apparatus
}

\author{
A. Gilat ${ }^{1, a}$, and T.A. Matrka ${ }^{1}$ \\ ${ }^{1}$ Ohio State University, Department of Mechanical Engineering, 201 W. 19th Ave., Columbus, OH, \\ 43210 USA
}

\begin{abstract}
A new apparatus for testing in compression at intermediate strain rates is introduced. The apparatus consists of a loading hydraulic actuator and a very long (40 m) transmitter bar. The specimen is placed with one end touching the end surface of the long bar and the other end free. The specimen is loaded by the actuator that impacts the specimen's free end directly. Once loaded, the specimen deforms between the actuator and the transmitter bar. As the specimen is loaded and deformed, a compression wave propagates into the transmitter bar. The amplitude of this wave is measured with strain gages that are placed on the transmitter bar at a distance of about five diameters from the specimen. The wave in the transmitter bar propagates all the way to the end of the bar and then reflects back toward the specimen. The experiment can continue until the reflected wave reaches the strain gages (milliseconds). The strain in the specimen (full field) is measured directly on the specimen using Digital Image Correlation with high speed cameras.
\end{abstract}

\section{Background}

The basic mechanical properties of materials (stress strain relation and failure) are typically determined by testing material coupon specimens in tension, compression, and shear. When the effect of strain rate on these properties is investigated, the tests are done at different strain rates. Standard hydraulic testing machines are usually used for testing at quasi-static strain rates in the range from $10^{-5} \mathrm{~s}^{-1}$ up to about $2 \mathrm{~s}^{-1}$. These tests are called quasi-static because the specimen and the testing machine are in static equilibrium during the test. At high strain rates, the split Hopkinson bar technique is used for testing at strain rates ranging from about $300 \mathrm{~s}^{-1}$ to about $5000 \mathrm{~s}^{-1}$. In this technique, the specimen is short and is in a state of equilibrium (inertia effects are not considered) during most of the test (except in the very beginning of the test). The rest of the apparatus (the bars) are not in static equilibrium. The stress waves that propagate in the bars are recorded and are used for determining the history of the deformation and stress in the specimen.

Tests at strain rates between about $10 \mathrm{~s}^{-1}$ and $200 \mathrm{~s}^{-1}$, defined here as intermediate strain rates, are difficult to conduct. They are too low to be done with the standard split Hopkinson bar technique, and as explained below, they are too high to be done with standard hydraulic machines because in this range inertia effects become significant. Many researchers have tried to conduct intermediate

\footnotetext{
a e-mail : gilat.1@osu.edu
}

This is an Open Access article distributed under the terms of the Creative Commons Attribution-Noncommercial License 3.0, which permits unrestricted use, distribution, and reproduction in any noncommercial medium, provided the original work is properly cited. 
strain rate tests with hydraulic machines. The actuator of a typical machine can move fast enough to deform the specimen at the required strain rate. Sometimes the hydraulic machines use an open loop control with an actuator that has a slack. In this way the actuator accelerates to the required speed before it get engaged with the specimen. The results from such tests, however, are not accurate. The problem is that during the test the whole machine is not in static equilibrium and stress waves and inertia effects cannot be handled by the machine. The time scale of an intermediate strain rate test is of the same order as the time scale for the stress waves to travel in the frame of the machine and the time it takes for the various components of the machine (grips, load cell, mechanical connections) to accelerate. The records from intermediate strain rate tests done with a hydraulic machine (force measured by the load cell and strain, if measured) are typically noisy with large oscillations (referred to as ringing) [1]. The problem is that the force that is measured by the load cell (assuming that the frequency response of the load cell itself is suitable), that is typically placed at the top of the machine, is not exactly the force that is applied to the specimen at the same instant. As the specimen is loaded, different components of the testing machines (grips, adaptors, etc.) accelerate at different rates (depending on their mass and geometry) and the reading of the load cell includes the combined inertia effect of all the components.

In many cases the noisy records from an intermediate strain rate test are smoothed by electronic or numerical means and the smoothed stress strain curves are considered to represent the property of the tested material. Attempts have been made to reduce the noise and oscillations in the records in such tests and/or to account for the inertia effects, [2-5]. For example, in addition to the machine load cell, stiff load cells (quartz based) have been placed close to the specimen. The intention is to measure the force that is actually applied to the specimen. Unfortunately, the records are still noisy with ringing due to wave reflections from different components of the machine. In other experiments, an accelerometer is attached to the grips of the specimen. The intention is to multiply the signal from the accelerometer by a constant and add it to the signal of the load cell. In some cases this can reduce the noise, but due to the complexity of inertia effects and wave reflections it still does not give good results. In a different approach the whole testing machine is modelled (including dynamic effects and waves) by assuming a constitutive relationship for the specimen that is tested. The simulation predicts the noisy record of the load cell and if it agrees with the measured record it is concluded that the assumed response of the specimen is the actual property of the material that is tested.

The present paper introduces a new apparatus for testing materials in compression at strain rates between about $10 \mathrm{~s}^{-1}$ and $200^{-1}$. The apparatus consists of a hydraulic actuator and a long transmitter bar. Stress strain curves obtained from testing specimens made of epoxy are clean and smooth without ant evidence of oscillations or ringing.

\section{Experimental technique}

The new compression intermediate strain rate apparatus, shown schematically in Fig. 1, is made of a hydraulic actuator and a long transmitter bar. The transmitter bar and the impact face of the actuator are both made from materials that remain elastic during the test. The specimen is placed with one end touching the end surface of the long bar and the other end free. The specimen is loaded by the actuator that impacts the specimen's free end directly. Once loaded, the specimen deforms between the actuator and the transmitter bar. As the specimen is loaded and deformed, a compression wave propagates into the transmitter bar. The amplitude of this wave is measured with strain gages that are placed on the transmitter bar (at a distance of about five diameters from the specimen). The transmitted wave is used for measuring the force that is applied to the specimen (as in the compression split Hopkinson bar). The wave in the transmitter bar propagates all the way to the end of the bar and then reflects back toward the specimen. The experiment can continue until the reflected wave reaches the strain gages. Since the strain rates are relatively low, the duration of the test is relatively long (milliseconds) and the transmitter bar has to be quite long. The experiment has to be designed (cross-sectional areas of the specimen and the transmitter bar) such that the amplitude 
of the stress wave in the transmitter bar is relatively small. The actuator must be large enough (load capacity) such that it moves at essentially constant speed throughout the duration of the test. The strain in the specimen during the tests is measured by 3D Digital Image Correlation (DIC). The DIC is used for measuring the strain directly on the specimen, and for determining the engineering strain from measuring the difference between the displacement of the end of the transmitter bar and the face of the actuator.

The actual apparatus is shown in Figs. 2-4. The transmitter bar is made of $40 \mathrm{~m}$ long $22.23 \mathrm{~mm}$ diameter stainless steel bar. The hydraulic actuator was custom made for the present application, and can move at a speed of $2 \mathrm{~m} / \mathrm{s}$ while applying a force of up to 22,000 N.

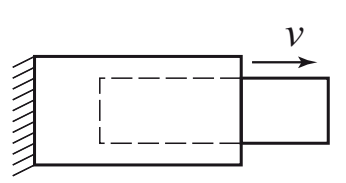

Actuator

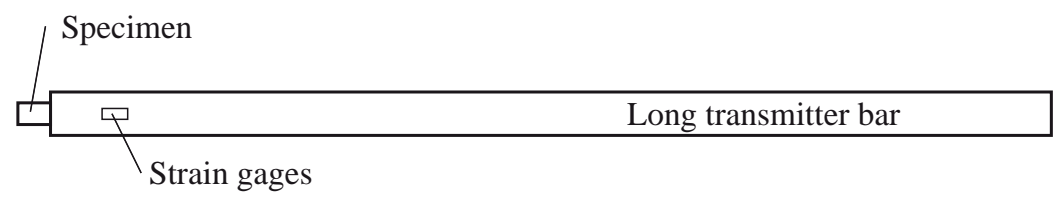

Fig. 1. Schematics of the compression intermediate strain rate testing apparatus.

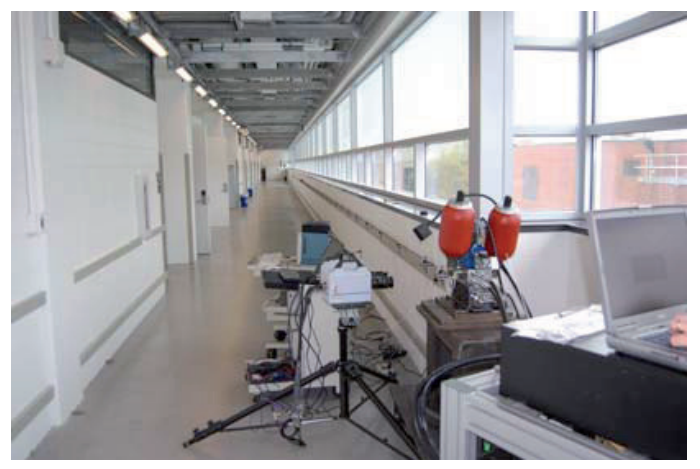

Fig. 2. Overall view of the compression intermediate strain rate testing apparatus.

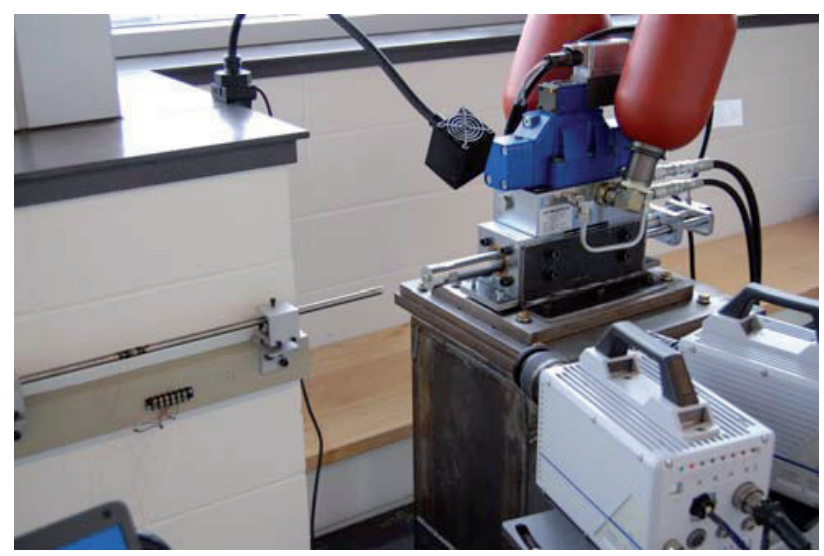

Fig. 3. Hydraulic actuator and transmitter bar of the compression intermediate strain rate testing apparatus. 


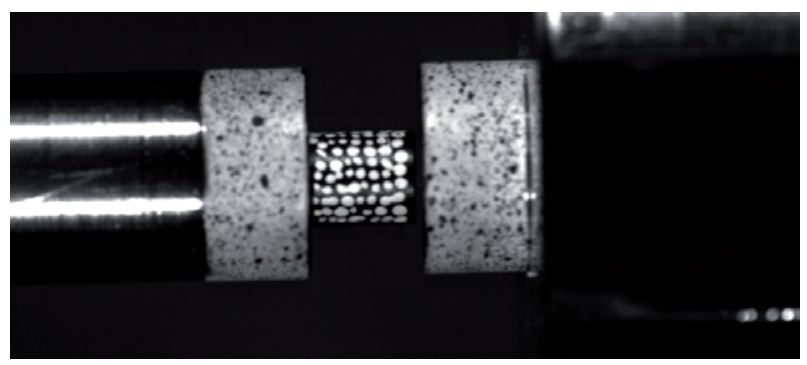

Fig. 4. Specimen between the actuator and the transmitter bar.

\section{Results}

Application of the new compression intermediate strain rate apparatus is shown here for testing specimens made of PR-520 epoxy. The force recorded by the strain gages on the transmitter bar during a test is shown in Fig. 5. The curve in this figure is the raw signal that was recorded (multiplied by a constant). The signal was not changed (averaged, or smoothed) by any electronic or numerical means. As can be seen, the signal is clean and smooth without any oscillations. The strain measured by the DIC system is shown in Fig. 6. The figure shows the Lagrange strain measured on the specimen directly. The strain is an average strain determined by DIC over most of the area of the specimen. Figure 6 shows also the engineering strain determined from the relative displacement between the ends of the specimen divided by the initial length of the specimen. The relative displacement is determined by applying DIC to the end of the transmitter bar where the specimen is placed and to the end of the actuator that impacts the specimen. As can be seen in Fig. 6, the strains determined by the two methods are essentially identical at small strains up to about strain of 0.1 . Later on as the strains become larger the Lagrange strain is smaller than the engineering strain. The strain rate is obtained from the slope of the strain vs. time plot. From Fig. 6 the strain rate is approximately $80 \mathrm{~s}^{-1}$. The stress stain curve from is test is shown in Fig. 7.

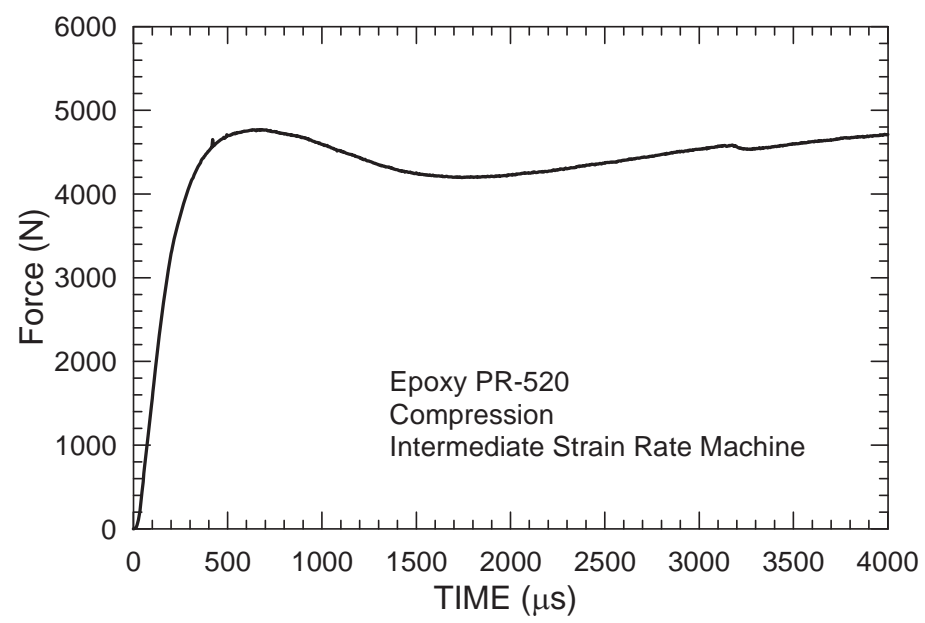

Fig. 5. Force measured on the transmitter bar. 
14th International Conference on Experimental Mechanics

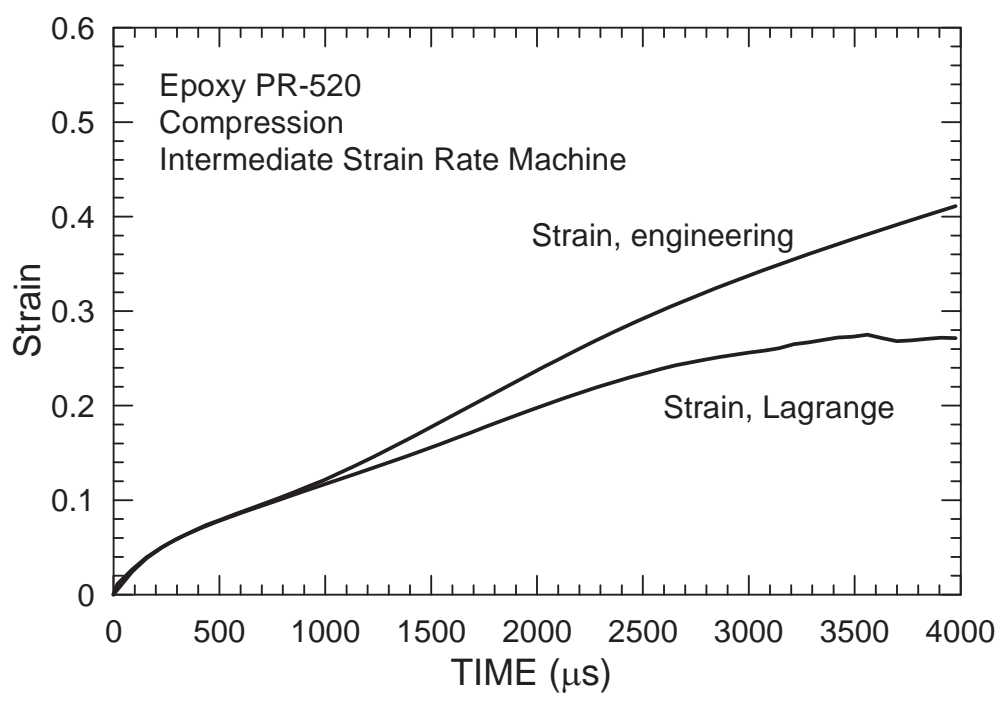

Fig. 6. Strain measured using DIC.

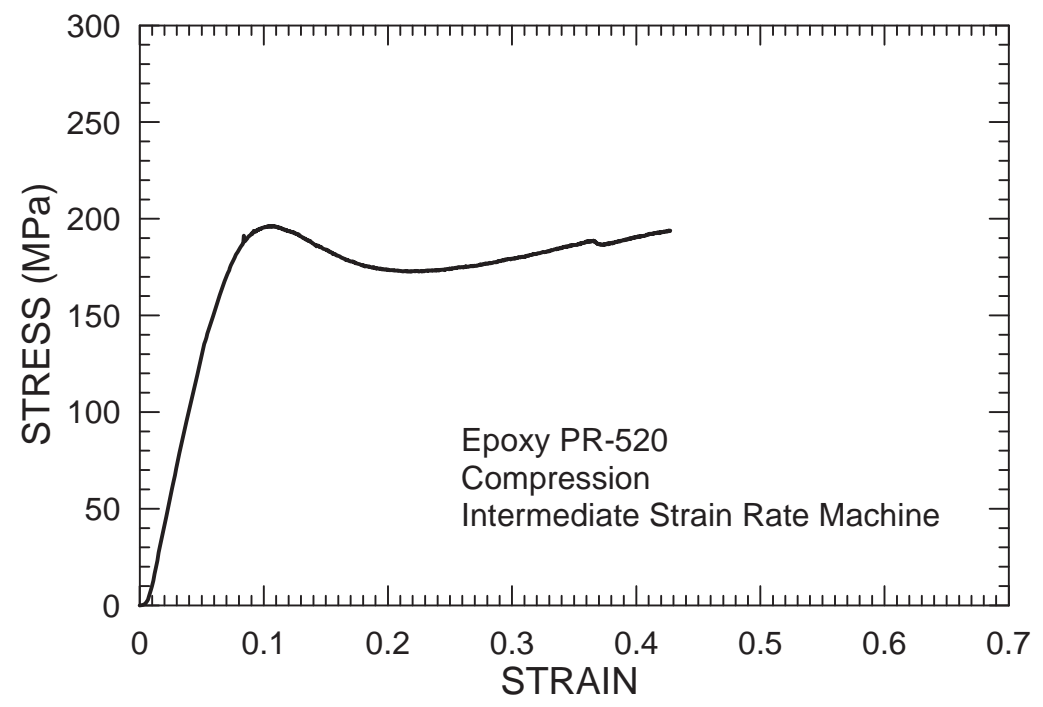

Fig. 7. Engineering stress strain curve of epoxy PR-520 at strain rate of approximately $80 \mathrm{~s}^{-1}$.

\section{Conclusions}

A new apparatus for compression testing at intermediate strain rates ranging from about $10 \mathrm{~s}^{-1}$ to 200 $\mathrm{s}^{-1}$ has been presented. The apparatus consists of a hydraulic actuator that presses the specimen against a long transmitter bar. The stress in the specimen is determined from the amplitude of the compression wave in the transmitter bar. The strain is measured with digital image correlation using high speed cameras. The stress strain curves that are obtained from tests with this apparatus are smooth without any oscillations (ringing) that are typical in tests at these strain rates with standard hydraulic machines. 


\section{Acknowledgements}

The research reported in this paper was supported by NASA (NRA Grant NNX08AB50A). Many thanks are due to the project manager, Dr. Mike Pereira of NASA Glen Research Center.

\section{References}

1. B.L. Boyce, M.F. Dilmore, Int. J. Impact Engineering, 36, 263 (2009)

2. H.S. Shin, H.M Lee, M.S. Kim, Int. J. Impact Engineering, 24, 571 (2000)

3. W. Bleck, I. Schael, Steel Res. 171 (5), 173 (2000)

4. S. Diot, D. Guines, A. Gavrus, E. Ragneau, J. Impact Engineering, 34, 1163 (2007)

5. R. Othman, P. Guegan, G. Challita, F. Pasco, D. LeBreton, Int. J. Impact Engineering, 36, 460 (2009) 